CIENCIA Y SOCIEDAD

Volumen XXXI, Número 1

Enero-Marzo 2006

\title{
ESTUDIO DE SEGUIMIENTO EGRESADOS Y EGRESADAS CON HONORES ACADÉMICOS DEL INTEC 1978-2004
}

\section{Evelin Cáceres Castellanos*}

\section{RESUMEN}

Se presentan los principales hallazgos del estudio de seguimiento realizado a los egresados con honores académicos del Intec de las promociones 1978 al 2004.

La investigación incluyó una encuesta a través de la cual se logró dar seguimiento a $1,219(51 \%)$ de los egresados con honores del período, la cual se complementó con dos sesiones de grupos de enfoque que se llevaron a cabo a fin de profundizar en algunos aspectos claves del estudio.

Se analizan factores concernientes a situación laboral, estudios de grado en el Intec, continuación de los estudios y desarrollo profesional de los egresados con honores académicos; así como su evaluación sobre los puntos neurálgicos de la universidad y sus sugerencias para la misma.

PALABRAS CLAVES

Egresados, honores académicos, Intec, datos laborales, continuación estudios.

\section{ABSTRACT}

We present the results of a research about the following of graduates with academic honors from INTEC University, during the period from 1978 to 2004.

The research included a survey which allowed the study of $1,219(51 \%)$ of the graduates with honors from this period. The same was completed with two focused group sessions that were made with the purpose of having a careful study of some key aspects of the research.

Some factors are analyzed concerning labor situation, degree studies at Intec, further education, and professional development of graduates with academic honors; as well as the evaluation of neuralgic points of the university procedures.

* Encargada de Relaciones con Egresados de la Universidad INTEC Email: ecaceres $(a$ intec.edu.do 


\section{KEY WORDS}

Graduates, academic honors, Intec, studies continuation.

\section{Introducción}

El Estudio de Seguimiento a Egresados con Honores Académicos del INTEC, es una iniciativa del Departamento de Desarrollo y Egresados, a través de su Oficina de Relaciones con Egresados. El mismo, fue presentado en la segunda convocatoria del año 2004 del Concurso de Estipendios para Investigaciones de la División de Investigaciones y Extensión del INTEC, resultando seleccionado para su realización.

Con el fin de poder ampliar el alcance de la investigación y que la misma pudiera abarcar la mayor cantidad de egresados posible, se presentó una propuesta de co-patrocinio al Grupo E. León Jiménes, en el marco de la colaboración que el INTEC y tan prestigiosa entidad han establecido a lo largo de los años; accediendo gentilmente el Grupo León Jiménes a acompañarnos en el desafío de realizar el primer estudio que de esta naturaleza se tenga cuenta en el país.

\section{Objetivos del Estudio}

El estudio se diseñó con el fin de dar seguimiento a los 2,378 egresados de las promociones 1976 al 2004 que han obtenido honores académicos en el Intec (los cuales representan el 16\% del total de egresados). A través del mismo se abordaron aspectos tales como:

- Situación laboral actual.

- Continuación de estudios de especialización.

- Otros honores académicos y/o reconocimientos recibidos a posteriori.

- Fortalezas y debilidades que a su juicio posee la universidad, en los tres ámbitos: docencia, investigación y extensión; a partir de su experiencia como estudiante.

- Se obtendrán sugerencias y recomendaciones para la universidad, dirigidas al fortalecimiento de esta última con sus egresados y el sector productivo nacional.

Con los hallazgos arrojados por esta investigación se pretende:

- Sentar un precedente para próximos estudios sobre la totalidad de nuestros egresados. 
- Dotar a la Comunidad Académica de información clave sobre sus egresados, a fin de fortalecer su vinculación con los sectores productivos de la sociedad dominicana.

- Conocer la situación actual de nuestros egresados que obtuvieron honores académicos en los ámbitos: laboral, socio-económico y del nivel educativo alcanzado.

- Fortalecer las relaciones y vinculación de la universidad con sus egresados, de modo que se garantice su participación activa en las diferentes iniciativas y actividades que auspicia el INTEC.

\section{Aspectos Metodológicos}

Para lograr una mejor comprensión de las variables a estudiar, se optó por adoptar un diseño de investigación que combinara técnicas tanto cuantitativas como cualitativas.

En una primera fase, se realizó una encuesta, a cargo de la Encargada de Relaciones con Egresados del Intec, Lic. Evelin Cáceres. A través de este estudio se logró dar seguimiento a 1,219 de los egresados del INTEC de las promociones 1976 al 2004, que han obtenido honores académicos; lo que representa aproximadamente el $51 \%$ de la población en estudio.

El cuestionario se estructuró de manera que incluyera preguntas destinadas a obtener data sobre temas claves relacionados con datos personales y laborales, información acerca de sus estudios en el Intec, valoración de los principales aspectos del quehacer universitario, continuación de los estudios, valoración del impacto de haber obtenido honores académicos, logros personales y profesionales, así como las sugerencias de nuestros egresados para el Intec.

El trabajo de campo se llevó a cabo en el período comprendido entre el 15 de abril y el 21 de septiembre del año 2005. Dada la naturaleza del estudio y el hecho de que se planea a mediano plazo dar seguimiento a la totalidad de los egresados del Intec, se tomó la decisión de hacer un censo, intentando contactar y obtener respuesta de cada egresado, en vez de seleccionar una muestra aleatoria. Para la aplicación del cuestionario se utilizaron varias vías: telefónica ( $51 \%$ de los encuestados); internet ( $48 \%$ ) y personal ( $1 \%$ restante).

Para apoyar la difusión del estudio, se formuló una campaña de promoción de la investigación que incluyó varios medios: correo electrónico, prensa, visitas a distintos medios de comunicación en radio y televisión 
y material promocional, el cual se colocó en el campus y se envió a las principales empresas del país con el fin de que a través de los departamentos de Recursos Humanos se difundiera el estudio entre los egresados con honores que pudieran estar laborando en las mismas.

Para complementar el estudio, en una segunda etapa, se realizaron dos (2) grupos focales. Esta técnica consiste en reunir de ocho (8) a diez (10) personas con características homogéneas y preestablecidas, de acuerdo con los objetivos del estudio. Con esta herramienta de análisis cualitativo, se logró obtener una mayor comprensión y una visión más profunda de lo que es el perfil de nuestros egresados y sus puntos de vista sobre el Intec, la educación recibida en la universidad y sus ideas y sugerencias desde su rol de egresados para el fortalecimiento de la misma.

La realización de las sesiones estuvo a cargo de la reconocida firma Horwath Consulting, quienes en un gesto de identificación con el Intec y con la importancia que este estudio reviste para la educación superior del país, realizaron una valiosa contribución para la realización de las mismas. Las sesiones fueron dirigidas por la Lic. Sarah Aguirre, Directora de Investigación de Mercados y docente del Intec y se llevaron a cabo los días 8 y 9 de septiembre del presente año, contando con la participación de 20 de nuestros egresados con honores.

\section{Principales Hallazgos del Estudio}

En el marco del mes aniversario del Intec, hemos querido hacer de dominio público los hallazgos preliminares de este estudio sin precedentes en materia de educación superior en la República Dominicana; como una manera de que los resultados del mismo sean conocidos no sólo a lo interno sino que también sirvan como una fuente de referencia en el sector educativo a nivel nacional.

Los resultados de la investigación apuntan a una importante serie de conclusiones que demuestran el impacto positivo que nuestros egresados atribuyen al estudiar en el Intec. Los hallazgos reflejan el hecho de que los egresados con honores de nuestra universidad han pasado por nuestras aulas habiendo experimentado un crecimiento a nivel profesional y personal, del cual, ellos mismos dieron testimonio tanto en la investigación a nivel cuantitativo como a nivel cualitativo, como veremos más adelante.

Los principales hallazgos del estudio se presentan a continuación, estructurados en las siguientes temáticas: Datos laborales. Sobre sus estu- 
dios en Intec, Percepción del Intec Continuación de estudios, Desarrollo profesional y Sugerencias.

\section{Datos Laborales}

En este sentido, vale la pena destacar el posicionamiento que en el mercado laboral han tenido nuestros egresados con honores académicos. Así, encontramos que el $92 \%$ de ellos se encuentra empleado; resultando la tasa de desempleo de los mismos muy baja en comparación con la media nacional al 2004, que ascendía según fuentes oficiales a $18.4 \%$.'

Según los hallazgos, el sector privado contrata la mayor cantidad de nuestros egresados con honores (72\%); no obstante, el sector público sigue siendo una importante fuente de generación de empleos $(21 \%)$. Un $4 \%$ labora en instituciones sin fines de lucro e igual proporción de manera independiente.

Los egresados con honores contactados prestan sus servicios en unas 670 instituciones, pertenecientes a diferentes sectores productivos del país, entre los cuales podemos destacar: Educación (14\%), Banca (9\%), Telecomunicaciones (8\%) y Zonas Francas (7\%).

Las empresas donde laboran mayor cantidad de los encuestados son: Verizon (5\%), BHD (2\%), Grupo E. León Jiménes (2\%) y Banco Popular $(2 \%)$.

Un $89 \%$ de los que trabajan, ocupa cargos del nivel alto (38\%) o medio $(51 \%)$ en la jerarquía empresarial.

En cuanto a nivel salarial se refiere, un 58\% indicó percibir ingresos mensuales superiores a los RD $\$ 30,000$; mientras que sólo un $4 \%$ devenga menos de RD $\$ 10,000$.

El hecho de que el $76 \%$ realice trabajos relacionados directamente a la carrera estudiada en el Intec, nos hace inferir que la preparación académica lograda en la universidad, les permitió adquirir los conocimientos y destrezas necesarias para posicionarse en sus respectivas áreas.

Es importante señalar que en materia de equidad de género, los hallazgos nos permiten afirmar que en los 33 años de existencia del Intec, la proporción de egresadas (sexo femenino) se ha visto incrementada con-

1 Fuente: Encuesta Nacional de Fuerza de Trabajo, 2004, Departamento de Cuentas Nacionales y Estadísticas Económicas, División de Encuestas. 
siderablemente, como lo evidencia el hecho de que nuestras egresadas representen el $61 \%$ de los graduados con honores contra el $39 \%$ de los hombres. Esto concuerda con los planteamientos que señalan que cada vez más dominicanas están teniendo acceso a una educación a nivel superior.

Desafortunadamente, tal como lo reflejan los resultados, aún existe inequidad en lo que a ingresos y nivel de mando se refiere, cuando comparamos el posicionamiento de las egresadas versus el de los egresados. Mientras que el $59 \%$ de los egresados devenga ingresos mensuales superiores a los RD \$50,000; las egresadas que caen en ese rango representan apenas el $23 \%$ y mientras el $43 \%$ de ellos ocupa cargos de alto nivel, sólo un $29 \%$ de ellas se encuentra en ese eslabón de la jerarquía laboral.

\section{Sobre sus estudios en Intec}

A través del estudio se logró dar seguimiento a egresados con honores académicos de cada una de las 15 carreras que componen la oferta académica a nivel de grado del INTEC; siendo las más frecuentes en la muestra: Ingeniería Industrial (20\%), Administración de Empresas ( $14 \%$ ) y Educación Básica (12\%). (Ver gráfico 1)

En el gráfico 2 se puede observar la proporción se egresados por sexo en cada una de las carreras, llamando la atención el hecho de que en las carreras de Psicología, Educación Básica, Mercadeo y Contabilidad, entre el $80 \%$ y el $94 \%$ de los egresados son del género femenino.

Por su parte, en las carreras de Filosofía, Ingeniería Eléctrica, Mecánica y Electrónica predomina el género masculino en porcentajes que van del $86 \%$ al $100 \%$. En el caso de la carrera de Filosofía, es importante destacar que esta carrera se oferta en coordinación con el Instituto Filosófico Pedro F. Bonó y es impartida en su mayoría a seminaristas, lo que justifica el hecho de que la totalidad de sus egresados sean del género masculino. 


\section{Gráfico 1- Composición de la Muestra por Carrera de Grado}

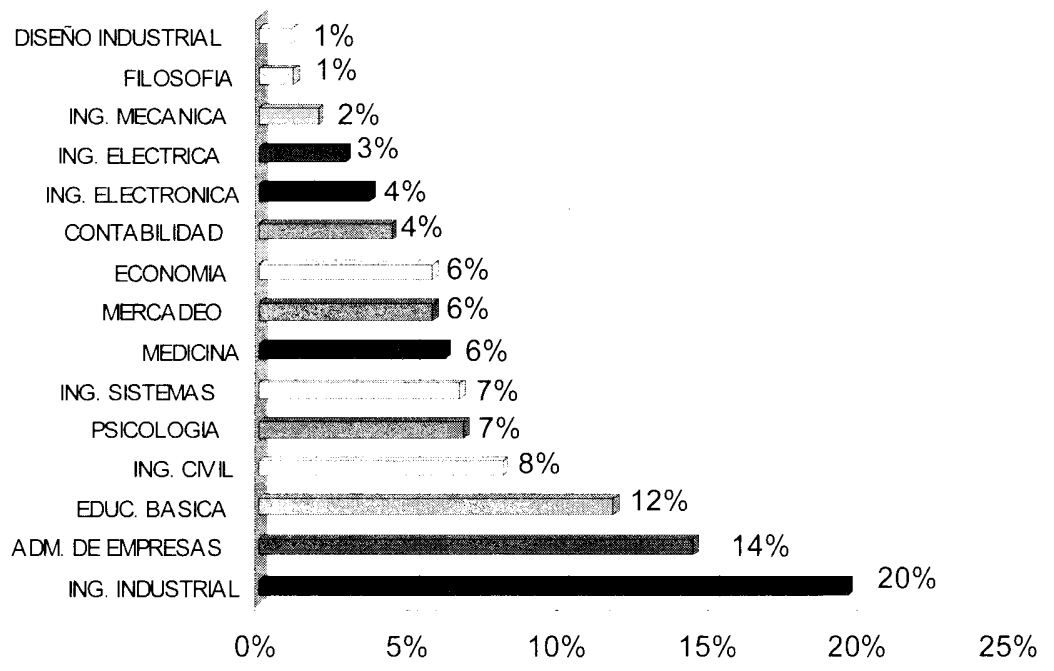

Fuente: INTEC, Estudio de Seguimiento Egresados con Honores Académicos, 1978-2004

Gráfico 2-Sexo de los encuestados por carrera

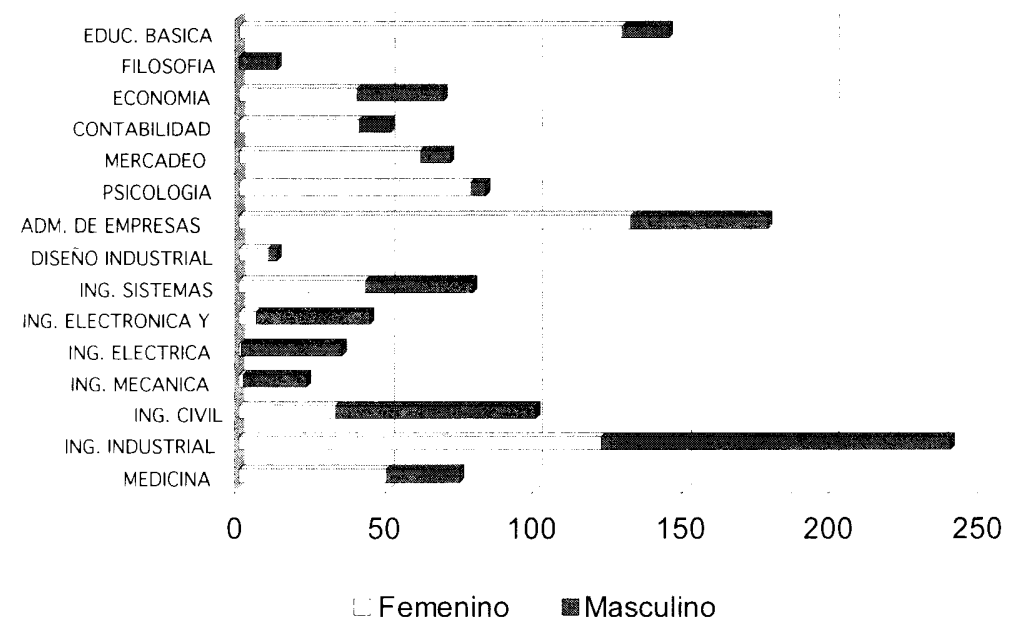

Fuente: INTEC, Estudio de Seguimiento Egresados con Honores Académicos, 1978-2004 
El gráfico 3 nos muestra la distribución de la muestra según los honores recibidos en Intec, a saber: Summa, Magna y Cum Laude.

\section{Gráfico 3- Honor Académico Obtenido}

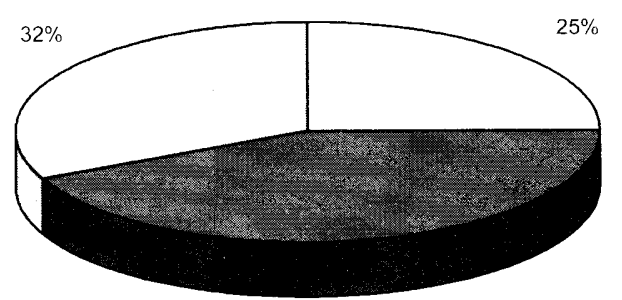

$43 \%$

$\square$ summa Cumlaude $\square$ magna cumlaude ramlaude

Fuente: INTEC, Estudio de Seguimiento Egresados con Honores Académicos, 1978-2004

Los egresados encuestados pertenecen a las promociones comprendidas entre el 1978 y el 2004; siendo el 65\% de las promociones del 1999 al 2004. (Ver gráfico 4)

\section{Gráfico 4- Año de Graduación}

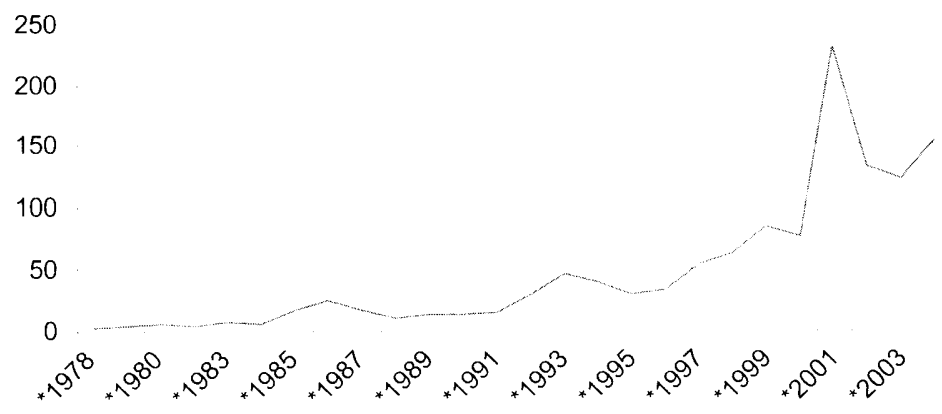

Fuente: INTEC, Estudio de Seguimiento Egresados con Honores Académicos, 1978-2004

El $75 \%$ de los encuestados tenía entre 20 y 23 años al momento de graduarse; siendo la edad promedio: 24 años y medio. La tendencia general fue la el graduarse al contar con 21 años de edad. Este dato nos permite hacer referencia al impacto del sistema trimestral de la universidad y al hecho de que nuestros estudiantes finalizan sus carreras en el tiempo pre- 
visto, constituyéndose de esta manera el Intec en una de las principales fuentes generadoras de jóvenes talentos que se insertan a la sociedad.

Un dato importante lo constituye el que el $52 \%$ de los egresados con honores recibieron el apoyo de los programas de becas y crédito educativo que ofrece la universidad; lo que evidencia el papel activo que juegan estos programas institucionales en contribuir a que cada vez más jóvenes, independientemente de su status económico, tengan acceso a una educación superior de calidad. El $21 \%$ de nuestros egresados con honores fue becado por el Programa Intec con los Estudiantes Sobresalientes (PIES), programa que a lo largo de los años ha contado con el patrocinio de diversas empresas e instituciones; $12 \%$ financiaron sus estudios a través del crédito educativo que ofrece la universidad, mientras que el 19\% resultó beneficiado con otra beca (destacándose las de la Secretaría de Educación $-5 \%$, las del Banco Central $-5 \%$ y las del BID - $2 \%$ ).

\section{Percepción del Intec}

La percepción que del Intec tienen sus egresados con honores es bastante positiva, como lo confirma la disposición del $95 \%$ de volver a seleccionar la universidad en el caso de que tuviera la oportunidad. Las razones por las cuales volverían al Intec se muestran en el gráfico 5.

\section{Gráfico 5-Razones para seleccionar nuevamente INTEC}

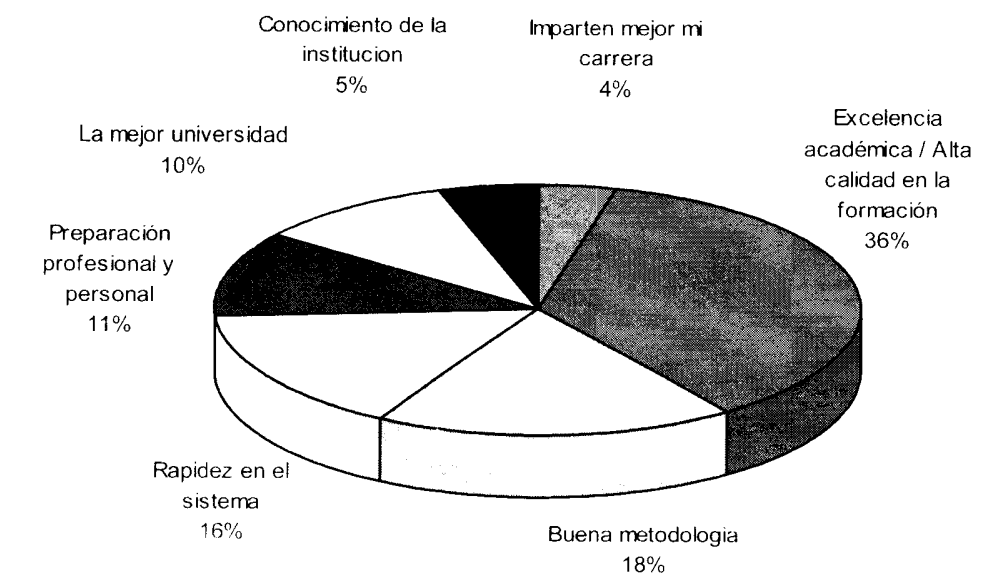

Fuente: INTEC, Estudio de Seguimiento Egresados con Honores Académicos, 1978-2004 
El Intec es evaluado en términos generales como Excelente o Muy Bueno en $95 \%$ de los casos (como se observa en el gráfico 6).

\section{Gráfico 6- Calificación INTEC en General}

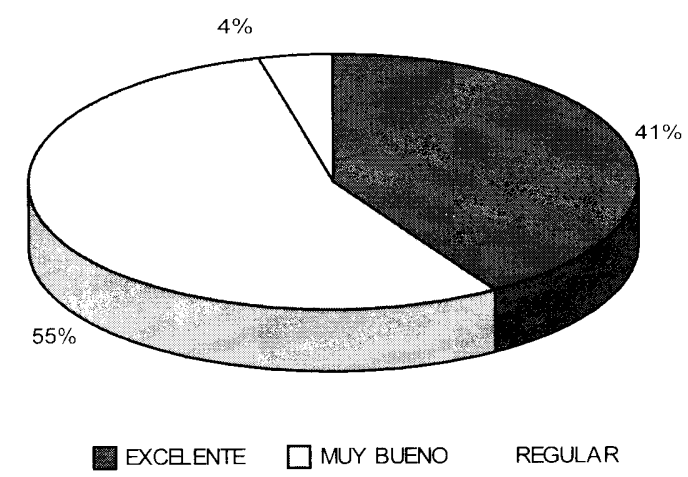

Fuente: INTEC, Estudio de Seguimiento Egresados con Honores Académicos, 1978-2004

En el mismo sentido, los egresados con honores calificaron varios aspectos del Intec, recibiendo mayor puntaje de "Excelencia": la Biblioteca (42\%) y los Programas Académicos (33\%). Por su lado, el cuerpo docente, instalaciones físicas, las áreas de Admisiones y Registro, las áreas académicas y los espacios para estudiantes en general son considerados como "Muy Buenos", siendo el Departamento de Registro, las Instalaciones Físicas y los espacios para la participación de los estudiantes, las áreas de mayor oportunidad. (Ver gráfico 7). Esto lo corroboran los participantes en las sesiones de grupos de enfoque, quienes además reconocen en la vinculación con los egresados, en especial en los últimos años, una nueva fortaleza de la universidad. 
Gráfico 7- Calificación Aspectos / Areas INTEC

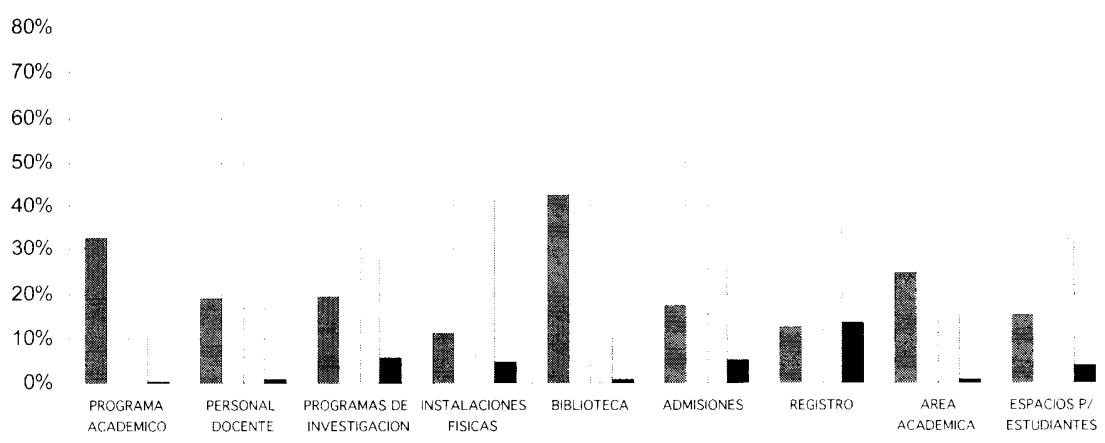

Fuente: INTEC, Estudio de Seguimiento Egresados con Honores Académicos, 1978-2004

En el estudio cualitativo, profundizamos en las razones que les hicieron optar por Intec a la hora de realizar sus estudios universitarios, citando las siguientes causas: la calidad, prestigio y estatus del que goza la universidad (destacándose sobre todo el área de Ingeniería); el sistema trimestral que garantiza el término de la carrera en un período relativamente menor en comparación con otras universidades; referencia de padres o terceros y las becas otorgadas para cursar sus estudios en el Intec.

Los participantes en estas sesiones reconocieron las ventajas de haber estudiado en Intec, mencionando por ejemplo, el haber adquirido una formación de calidad, el gozar del respeto de la sociedad, la facilidad para conseguir trabajo, la seguridad y el sentido de organización y responsabilidad adquiridos a su paso por la universidad. Ellos asocian el Intec a: progreso, calidad, practicidad, rapidez, dedicación, empeño, reto, lucha, esfuerzo, responsabilidad, disciplina y diferenciación.

En la encuesta, los egresados con honores concuerdan en el alto grado de pertinencia entre la formación recibida en el Intec y las necesidades que como profesionales han experimentado; manifestando el $85 \%$ que sus estudios en el INTEC fueron suficiente o bastante satisfactorios en ese sentido. (Ver gráfico 8) 
Gráfico 8-Medida de Respuesta de sus Estudios en el INTEC a sus necesidades como profesional
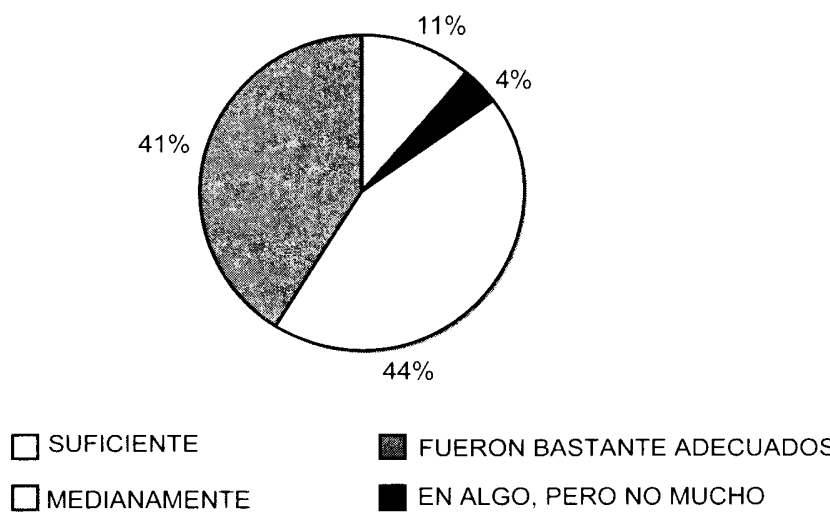

Fuente: INTEC, Estudio de Seguimiento Egresados con Honores Académicos, 1978-2004

El 69\% de nuestros egresados con honores dice estar en la disposición de participar en actividades de apoyo al Intec, lo que manifiesta la pertinencia de integrarlos de un modo más activo al quehacer universitario, con miras a lograr un fortalecimiento cada vez mayor a nivel institucional.

\section{Continuación de Estudios}

El 57\% de los encuestados reportó haber realizado estudios de especialización (52\% de los mismos a nivel de Maestría; 46\% Post-grado y $2 \%$ Doctorado). Este dato resulta bastante significativo cuando se toma en cuenta que estadísticas indican que sólo el $2 \%$ de todos los egresados del sistema educativo en la República Dominicana, logran realizar estudios de especialización.

Un 70\% de nuestros egresados con honores ha realizado sus especializaciones en el país, mientras que el restante $30 \%$ ha optado por universidades en el extranjero. El $56 \%$ de los que las han cursado en el país, indicó haberlo hecho en Intec, lo cual refleja la calidad atribuida a los programas ofrecidos por la universidad.

El alto porcentaje de egresados que manifestaron su interés en realizar estudios de especialización (77\%) y cursos de capacitación o actualización $(90 \%)$, representa un desafío para el Intec, de tratar de satisfacer esa demanda con una oferta curricular que dé respuesta en forma pertinente 
y con calidad a las necesidades de los mismos. En este sentido el estudio nos ha dado la oportunidad la identificación de los principales tópicos de interés de nuestros egresados con honores en materia de especializaciones y cursos de actualización, a fin de difundir entre ellos los programas con los cuales contamos y por otro lado, el diseñar nuevos programas que se ajusten a las necesidades aún no satisfechas.

\section{Desarrollo Profesional}

Encontramos que además de los honores académicos recibidos en el Intec, el $46 \%$ de nuestros egresados ha recibido otros reconocimientos por su destacado desempeño en los estudios a nivel medio (37\%) y superior $(16 \%)$; en el trabajo (34\%) y por su labor docente (19\%). Así mismo, el $15 \%$ reportó haber sido beneficiado por algún tipo de beca.

Sólo el 3\% de los egresados con honores está impartiendo docencia en la actualidad en el Intec, en contraste con el 57\% que está interesado en hacerlo. Es importante en este sentido el que la universidad desarrolle una estrategia de reclutamiento y selección de los mejores candidatos para incorporarlos a nuestro cuerpo docente. Uno de los productos del estudio es precisamente la identificación de esos potenciales docentes, de quienes estaremos enviando sus datos más importantes a las áreas correspondientes.

Resulta interesante constatar el interés de nuestros egresados con honores académicos en seguir innovando e incidiendo en un ejercicio profesional integral, al realizar el $83 \%$ de los mismos actividades como: la autoría de libros $(1 \%)$, la publicación de artículos en revistas profesionales o científicas o en la prensa (14\%), investigaciones (32\%), el haber fungido como ponente o expositor en alguna actividad (44\%) y el pertenecer a algún grupo profesional o gerencial (19\%).

\section{Sugerencias}

Las sugerencias de nuestros egresados para el Intec señalan la necesidad de crear conciencia y trabajar en un plan de mejoras en el cual ellos sean partícipes, a fin de lograr contrarrestar las áreas de oportunidad que ellos identifican; a saber: la calidad del cuerpo docente, la actualización continua de los programas académicos, de manera que respondan a las necesidades del sector laboral y la eficientización del sistema de selección de asignaturas. 
Por otro lado, para estrechar los vínculos universidad-egresado, ellos recomiendan la realización, frecuentemente, de actividades o reuniones de egresados, el mantener la comunicación constante con ellos, a través del correo electrónico, vía telefónica y otros medios a fin de mantenerlos al tanto de lo que ocurre en su Alma Mater y de la oferta académica de la misma a nivel de especialización y educación permanente.

El 7\% de los encuestados reconoció el esfuerzo que desde el año 2004 realiza el Intec, a partir de la creación de la Oficina de Relaciones con Egresados, impulsando iniciativas llamadas a lograr una mayor participación de nuestros egresados, recomendando continuar con las mismas.

En adición a todos los hallazgos citados, el estudio nos ha permitido actualizar los datos de este grupo de nuestros egresados; pudiendo en lo adelante fortalecer la participación de los mismos en nuestros programas a nivel de especialización y educación permanente; en los diversos proyectos estratégicos impulsados por su Alma Máter, tales como Fondo Conocimiento, Asociación de Egresados; así como el propiciar su integración en las instancias colegiadas, el cuerpo docente y el programa de investigaciones tecnológicas y científicas con los que cuenta la universidad.

Es el interés de la Oficina de Relaciones con Egresados el que las informaciones recopiladas a través de esta investigación impacten en el ejercicio de las funciones esenciales de nuestra Comunidad Académica, a fin de que sirva como reflexión sobre los puntos fuertes y débiles con los que, según los hallazgos cuenta la universidad a juicio de nuestros egresados con honores académicos, y por supuesto, de que se pueda tomar así algún tipo de acción en aquellos que representan áreas de oportunidad.

\section{Con los Resultados}

Ahora, estos resultados servirán como precedente para estudios de seguimiento bajo esta misma línea que realizaremos a la totalidad de nuestros egresados y nos ayudarán a fortalecer la relación empresa-sociedad-universidad. A través de Intec Honoris, actualizamos los datos de este grupo de egresados, lo que nos permitirá enviarles la oferta académica del Intec en programas de especialización y cursos de educación permanente, así como las convocatorias de becas y las oportunidades de trabajo que nos hacen llegar las empresas a través de nuestra Bolsa de Talentos, la cual 
hará especial énfasis en la colocación de aquellos egresados que no estén laborando.

Además, gracias al estudio, vamos a poder fortalecer la participación de nuestros egresados en las actividades que organicemos, propiciar su integración en las instancias colegiadas, el programa de investigaciones, la Asociación de Egresados y el proyecto Fondo Conocimiento.

Así mismo, podremos incorporar a nuestro cuerpo docente a aquellos egresados que manifestaron su deseo de ser parte de nuestro equipo y que cumplan con el perfil necesario para serlo. Por otro lado, realizaremos actividades de reconocimiento a egresados destacados en algún ámbito y publicaremos artículos sobre los mismos en los órganos de difusión del Intec.

Finalmente, remitiremos la lista de sugerencias de nuestros egresados con honores a las áreas competentes, a fin de que las mismas impacten en el ejercicio de las funciones esenciales de nuestra Comunidad Académica y sirvan de motor para la toma de decisiones encaminadas a mejorarlas.

\section{Bibliografía}

1 Aquino Fernández, Clary. "Estudio sobre el impacto del Programa Intec con los Estudiantes Sobresalientes (PIES)"/ Oficina de Desarrollo y Egresados. INTEC. 1997.

2 Cattafesta, Catherine. "Los Egresados u Egresadas del INTEC". Revista Ciencia y Sociedad. Vol. XXVII. No. 2. abril-junio 2002.

3 "Fulbright Faculty Development, Ecology, and Cost-Share Programs: A Survey of Alumni"/ LASPAU. Marzo, 2004. 48 p.

4 Instituto Tecnológico de Santo Domingo. "Programa de Consolidación y Expansión: Evaluación a posteriori Préstamo BID-INTEC 681//SF-DR". 1994. p. $12.28-34$

5 Kinnear, Thomas C. y James R. Taylor. "Investigación de Mercados: un enfoque aplicado". Mc. Graw Hill. 4ta Ed. México, 1997. 812 p.

6 Malhotra, Naresh K. "Investigación de Mercados: Un Enfoque Práctico". Pearson Educación. 2da. Ed. México, 1996. 890 p.

7 Perdomo de Dávalos, Rosalina."Estudio de seguimiento de los Egresados del Instituto Tecnológico de Santo Domingo de la Primera Década: 1975-1984". Facultad de Ciencias y Humanidades, Intec. 1986. 
Evelin Cáceres Castellanos: Estudio de seguimiento egresados y egresadas...

8 "Proyecto: Estudio de Seguimiento e Impacto de Egresados"/ Universidad de Córdoba. 2004.<http:unicordoba.edu.do.co/unicor/egresados/proyegresados. pdf $>$

9 Reglamentos académicos / Instituto Tecnológico de Santo Domingo.- 10. ed. - Santo Domingo, 1994. 53 p.

10 Soria Nicastro, Oscar y Berta Garibay. "Estudio de Seguimiento de Egresados: Disposiciones deseables y diseño curricular". Revista Nueva Época. Vol. 4. No. 7. enero-junio 2000. 